\title{
The microglial activation profile and associated factors after experimental spinal cord injury in rats
}

This article was published in the following Dove Press journal:

Neuropsychiatric Disease and Treatment

\section{Yuan Zhou' \\ Ning $\mathrm{Li}^{2}$ \\ Lin Zhu ${ }^{3}$ \\ Yixing $\operatorname{Lin}^{3}$ \\ Huilin Cheng' \\ 'Department of Neurosurgery, Jinling Hospital, Jinling School of Clinical Medicine, Nanjing Medical University, Jiangsu, China; ${ }^{2}$ Department of Surgery, Li Ka Shing Faculty of Medicine, The University of Hong Kong, Queen Mary Hospital, Hong Kong SAR, China; ${ }^{3}$ Department of Neurosurgery, Jinling Hospital, Jiangsu, China}

Correspondence: Huilin Cheng Department of Neurosurgery, Jinling Hospital, Jinling School of Clinical Medicine, Nanjing Medical University, 305 East Zhongshan Road, Nanjing, Jiangsu, China

Tel +862580860123

Fax +862551805396

Email huilin_cheng@yahoo.com
Background: Spinal cord injury (SCI) has imposed a great impact on the quality of life of patients due to its relatively young age of onset. The pathophysiology of SCI has been proven to be complicated. Microglia plays an important role in neuroinflammation and second injuries after SCI. Different environment and other factors may determine the microglial activation profile and what role they play. However, neither accurate time-course profiles of microglial activation nor influence factors have been demonstrated in varied SCI models.

Methods: A rat compressive SCI model was used. Microglial activation profile and contents of inflammatory factors including IL-1 $\beta$, IL- 6 and TNF- $\alpha$ were detected. Myelination status as well as levels of iron and glutamate concentration, adenosine triphosphate (ATP) and potassium are also assessed.

Results: Our results showed that the activated microglia participating in immune-mediated responses peaked at day 7 post SCI and gradually decreased during the following 3 weeks. Contrarily, myelination and oligodendroglia showed an opposite trend, indicating that microglia may be a key factor partly through inflammatory reaction. Iron and glutamate concentration were found to be the highest at day 7 after SCI while both ATP and potassium reached a low valley at the same time.

Conclusion: These findings showed a microglial activation profile and the alterations of associated factors after experiment SCI model. Moreover, our data suggest that high iron and glutamate concentration may be released by damaged oligodendroglia and contribute to the activation of microglial after SCI.

Keywords: spinal cord injury, microglia, oligodendroglia, iron, glutamate

\section{Introduction}

Spinal cord injury (SCI) is a serious neurologic disease requiring more effective treatments. The incidence of SCI is varied and the data in Asia is 12-61 cases/ 1 million with a mortality rate of $5.8 \%-16 \%{ }^{1}$ These data are much higher than those in developed countries. ${ }^{2}$ Patients with SCI are often accompanied with severe physical activity disorders. ${ }^{3}$ Due to a relatively young age of onset, SCI not only has great impact on the quality of life of the patients but also brings great pressure to the social health system.

The pathophysiology of SCI includes myelin loss, axonal degradation, oxidative stress, inflammatory response, activation of various cytokines, cell death, etc. ${ }^{4-7}$ Previous research has demonstrated that oligodendroglia apoptosis has been observed after SCI and has led to the occurrence of chronic demyelination. ${ }^{8}$ Furthermore, microglia, widely located throughout the central nervous system (CNS), have also contributed to the mechanism of SCI. As the main mediator of the CNS's innate immune response, microglia play an important role in neuroinflammation and second injuries after nerve 
injury. ${ }^{9-11}$ Primary traumatic SCI can induce the release of reactive oxygen species (ROS) and inflammatory factors, triggering the activation of microglia. ${ }^{8}$ In recent years, it has been recognized that microglia play a dual role in traumatic brain and SCI. ${ }^{12,13}$ On the one hand, microglia can promote tissue recovery, but on the other hand, they also cause nerve degeneration. Different environment and other factors may determine the microglial activation profile and what role they play. However, accurate time-course profiles of microglial activation have not been demonstrated in varied SCI models. Abdanipour et $\mathrm{al}^{8}$ showed that microglia activation reached a peak at day 2 as compared to other days after SCI, whereas other reports observed the peak had been at day 7 post-injury. ${ }^{14}$ Thus, one purpose of our study was to evaluate the microglial activation profile using a rat compressive SCI model. Though the activation of microglial is influenced by many factors after injury, specific factors are far from clear. ${ }^{10,15}$ At certain concentrations, glutamate can induce microglial to activate, releasing pro-inflammatory factors. ${ }^{16}$ Iron, ATP and potassium content are vital factors related to immune response post injury. ${ }^{17-19}$ The other objective of our study was to explore factors affecting the activation of microglia.

\section{Materials and methods Animals}

A total of 108 male, adult Sprague Dawley rats (6-8 weeks) weighing between 230 and $270 \mathrm{~g}$ were purchased from Hua Fukang Biological Technology Co., Ltd, Beijing, China. They were housed on a 12-hour light/dark cycle at $23^{\circ} \mathrm{C} \pm$ $1^{\circ} \mathrm{C}$ with free access to food and water and were acclimatized for 1 week before the experiment began. All experiment protocols of this study including animal usages and the surgical procedures were approved by the Animal Care and Use Committee of Nanjing Medical University and were in accordance with the Guide for the Care and Use of Laboratory Animals by the National Institutes of Health (NIH).

\section{Groups and experimental design}

Rats were randomized into six experimental groups: 1) sham operation group $(n=18), 2) 1$ day after SCI group $(n=18)$, $3) 3$ days after SCI group $(n=18), 4) 7$ days after SCI group $(\mathrm{n}=18), 5) 14$ days after SCI group $(\mathrm{n}=18)$, and 6) 28 days after SCI group $(n=18)$. All the rats from the groups underwent an assessment of their motor function before injury and at varied days after the operation. Finally, all the spinal cords were harvested. In each group, six rats were for the Western blot analysis, another six for histologic staining and the other six for detection of pro-inflammatory cytokines and factors.
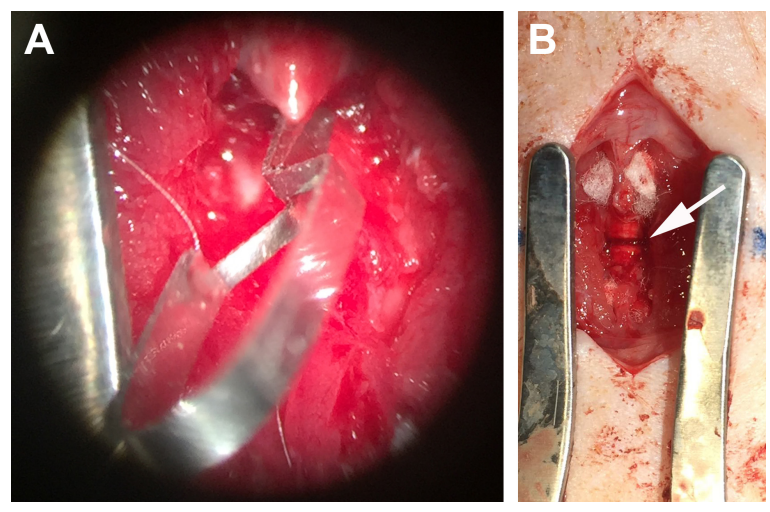

Figure I Compressive SCl model.

Notes: (A) Under an operating microscope, compressive injury was induced by a 2-minutes compression $\mathrm{SCl}$ using a $10 \mathrm{~g}$ aneurysm clip. (B) After removing the aneurysm clip, a clear imprint (white arrow) indicating the injury position can be seen.

Abbreviation: $\mathrm{SCl}$, spinal cord injury.

\section{Compressive SCI model}

Rats were firstly anesthetized with $10 \%$ chloral hydrate $(0.35 \mathrm{~mL} / 100 \mathrm{~g})$ through intraperitoneal injection. Half the quantity $(0.17 \mathrm{~mL} / 100 \mathrm{~g})$ was then injected every hour for continuous anesthesia throughout the entire experiment. Rats were placed in the prone position on a warming pad. All surgeries were performed by the same surgeon following the methods outlined previously. ${ }^{14,20}$ Briefly under aseptic conditions, the T10 spinal cord segment was fully exposed by T9-T11 dorsal laminectomies leaving the dura intact using an operating microscope (M500-N, Leica, Heerbrugg, Switzerland). Subsequently, compressive injury was induced by a 2-minute compression SCI using a $10 \mathrm{~g}$ aneurysm clip (5.0 mm*0.8 mm, Kent Scientific, Torrington, CT, USA; Figure 1). Attention was paid to whether the rats had a tail flicking and several quick twitches of double hind limbs followed by soft paralysis. The clip was carefully removed avoiding rotation and traction 2 minutes post clipping and the stratified incision was finally closed. The sham operation group only received the vertebral laminectomies to expose the spinal cord according to the above steps without clipping.

\section{Post-operative care}

All rats were allowed to recover in a warmed cage, and food and water was easily accessible. Animals were given postoperative analgesia ( $50 \mathrm{mg} / \mathrm{kg}$ buprenorphine) subcutaneously to prevent pain. Saline $(2 \mathrm{~mL})$ was given through subcutaneous injection immediately after the operation. The antibiotic gentamicin ( $5 \mathrm{mg} / \mathrm{kg}$, intramuscular) was also administered daily for the following 7 days or until the animals were euthanized. 


\section{Motor function assessment}

The Basso, Beattie, and Bresnahan (BBB) Locomotor Rating Scale was performed to assess the motor function of the rats before the operation, after the rats recovered from anesthesia completely, and at days $1,3,7,14$, and 28 post injury in the different groups. The BBB score ranges from 0 (no hindlimb movement) to 21 (normal motor function). ${ }^{21}$ Two blinded observers evaluated the score individually, and the average value of the two observers' scores was chosen.

\section{Spinal tissue preparation}

At days 1, 3, 7, 14, and 28 post injury, the rats in the SCI group and the sham operation group were anesthetized, and transcardially perfused with pre-cooled saline. For isolation of protein, the spinal cord containing the lesion area was carefully removed from the vertebrae column, immediately frozen in liquid nitrogen, and then transferred to a $-80^{\circ} \mathrm{C}$ freezer until use. For Luxol fast blue (LFB) staining and immunohistochemistry, the rats were perfused with the above saline followed by cold $4 \%$ paraformaldehyde. The injury site and adjacent spinal cord tissue were removed and immersed in 4\% paraformaldehyde overnight. For immunofluorescence, the specimens were subsequently immersed in $20 \%$ sucrose followed by $30 \%$ sucrose. For determination of pro-inflammatory cytokines and factors such as iron, glutamate, ATP and potassium, the injured areas of spinal cord were removed and carefully collected.

\section{Western blot analysis}

Firstly, the frozen samples were homogenized in extract buffer with protease inhibitors (PMSF) and centrifuged at $12,000 \times \mathrm{g}$ for 15 minutes at $4^{\circ} \mathrm{C}$. Then, protein concentrations were determined by the Bradford method. Equal amounts of protein per lane were separated by $10 \%$ sodium dodecyl sulfate-polyacrylamide gel electrophoresis and transferred to polyvinylidene difluoride (PVDF) membranes. The membranes were blocked for 2 hours in blocking buffer [Tris-buffered saline $/ 0.05 \%$ Tween 20 (TBST) containing $5 \%$ skim milk] and then incubated overnight at $4{ }^{\circ} \mathrm{C}$ with primary antibodies against Myelin basic protein (MBP) (1:1,000; Abcam, Cambridge, MA, USA) and $\beta$-actin (1:5,000; Bioworld Technology Inc., St Louis Park, MN, USA) in blocking buffer. After being washed with TBST ( $3 \times 10$ minutes), the membranes were incubated with goat anti-rabbit horseradish peroxidase-conjugated $\operatorname{IgG}(1: 5,000$; Bioworld Technology) for 2 hours at room temperature. Finally, the protein bands were visualized by enhanced chemiluminescence (ECL) Western blot detection reagents (EMD Millipore, Billerica, MA, USA). Band density was quantified using Un-Scan-It 6.1 software (Silk Scientific Inc., Orem, UT, USA).

\section{LFB staining}

Myelination of the spinal cord was evaluated via LFB staining. Briefly, spinal cord serial sections ( $5 \mu \mathrm{m}$ thick) were dehydrated in a gradient of ethanol and stained in $0.1 \%$ solvent blue 38 (Leagene Biotechnology Co., Ltd, Beijing, China) in acidified $95 \%$ ethanol overnight at $60^{\circ} \mathrm{C}$. After rinsing with $95 \%$ ethanol and distilled water, sections were then differentiated with $0.05 \% \mathrm{Li}_{2} \mathrm{CO}_{3}$ and $35 \%$ ethanol several times until the contrast between gray matter and white matter was clearly detected. The measurements were performed with the aid of ImageJ software (version 1.51; National Institutes of Health, USA). The mean integrated optical density (IOD) of positive areas in the six views was regarded as the data of each section. A total of four sections from each animal including two sections containing the epicenter of the lesion plus rostral area and the other two containing the epicenter of the lesion plus caudal area were used for quantification. The six random high-power fields $(400 \times)$ within each section were selected without overlap from white matter. The final average number of the four sections was regarded as the data for each sample. All the processes were conducted by two investigators blinded to the grouping.

\section{Immunohistochemistry}

Consecutive $4 \mu \mathrm{m}$-thick serial sections were cut and routinely deparaffinized. Endogenous peroxidase was blocked with 3\% $\mathrm{H}_{2} \mathrm{O}_{2}$ /methanol. Nonspecific antibody binding was blocked by incubating the sections in blocking buffer (10\% normal goat serum in PBS) for 30 minutes. Primary antibodies rabbit anti-MBP (1: 50; Abcam) and rabbit anti-Plastin L (LPL; 1: 100; Abcam) were applied overnight at $4^{\circ} \mathrm{C}$. After being washed three times in PBS for 5 minutes each, the sections were incubated with horseradish peroxidase-conjugated $\operatorname{IgG}$ (1:500; Santa Cruz Biotechnology, Inc., Dallas, TX, USA) for 60 minutes. 3,3-Diaminobenzidine (DAB) $/ \mathrm{H}_{2} \mathrm{O}_{2}$ solution was used for coloration. Finally, cell nuclei were counterstained with hematoxylin.

Six random nonoverlapping high-power fields $(400 \times)$ in each coronary section were chosen from white matter, and the mean IODs of the MBP/LPL-positive areas in the six views was regarded as the data of each section. A total of four sections from each animal used for quantification 
included two sections containing the epicenter of the lesion plus rostral area and the other ones containing the epicenter of the lesion plus caudal area. The final average number of the four sections was regarded as the data for each sample. All the processes were conducted using Image-Pro Plus software (Media Cybernetics, Rockville, MD, USA) by two investigators blinded to the grouping.

\section{Double immunofluorescence staining}

For double immunofluorescence, serial $5 \mu \mathrm{m}$ coronal sections were obtained using a cryostat. Four sections from each animal were collected using the same way of immunohistochemistry. Based on the established immunostaining protocol, the slides were incubated in blocking buffer (10\% normal goat serum in PBS containing $0.1 \%$ Triton $\mathrm{X}-100$ ) for 2 hours followed by overnight incubation at $4{ }^{\circ} \mathrm{C}$ in primary antibodies [rabbit anti-ED-1 $(1: 100 ; \mathrm{PL}$ Laboratories Inc., Richmond, BC, Canada); mouse antiMHC-II (1:100; Abcam)]. The next day, the slides were washed with PBS three times for 5 minutes and then incubated with appropriate secondary antibodies for 1 hour at room temperature. The slides were washed three times in PBS, counterstained with 4',6-diamidino-2-phenylindole (DAPI; Beyotime Biotechnology Inc., Nantong, China) for 2 minutes, and rinsed with PBS. Coverslips were applied with mounting media. Fluorescence was imaged on an Olympus IX71 inverted microscope system and analyzed using Image-Pro Plus 6.0 software (Media Cybernetics). The specificity of the immunofluorescence reaction was evaluated by replacing the primary antibody with PBS. The mean number of the activated microglial cells (ED-1/MHC-II double-stained cells) in the six views were regarded as the data of each section. The six random high-power fields $(400 \times)$ were chosen without overlap from white matter within each section. The final average number of the four sections was regarded as the data for each sample. All procedures were performed by two investigators who were blind to the experimental groups. Data were presented as the ratio of mean IODs of different SCI group to sham operation group.

\section{Determination of iron and glutamate levels in the injured tissue}

Iron and glutamate levels in the injured tissue were measured using the Iron Assay Kit (Sigma-Aldrich Co., St Louis, MO, USA) and Glutamate Assay Kit (Sigma-Aldrich Co.). The injured areas of spinal cord were collected and weighed. Then, the tissue was rapidly homogenized in Iron
Assay Buffer or Glutamate Assay Buffer and centrifuged at $13,000 \times \mathrm{g}$ for 10 minutes at $4^{\circ} \mathrm{C}$. Next, the supernatant was collected and the following procedure was performed according to the manufacturer's instructions. The blank and sample tubes were measured three times and the absorbance values at $593 \mathrm{~nm}$ or $450 \mathrm{~nm}$ were recorded for analysis. Iron and glutamate levels were expressed as micromoles per gram of protein.

\section{Enzymatic method for determining ATP and potassium}

ATP Assay Kit (Jiancheng, Nanjing, China) and Potassium Assay Kit (Jiancheng) were performed to quantify the glutamate and potassium concentration in the injured tissue. First, collected injured areas of spinal cord were weighed, added nine times volume of deionized water, and homogenized. Second, protein precipitator was mixed. The third step was centrifugation at $1,200 \times g$ for 10 minutes. Finally, the supernatant was tested according to the manufacturers' instructions. The absorbance values at $636 \mathrm{~nm}$ or $450 \mathrm{~nm}$ were recorded for further reckoning. ATP and potassium concentrations were expressed as micromoles or millimoles per gram of protein.

\section{Enzyme-linked immunosorbent assay (ELISA)}

The frozen cortex tissue was mechanically homogenized in $1 \mathrm{~mL}$ of lysate buffer and centrifuged at $12,000 \times g$ for 20 minutes at $4^{\circ} \mathrm{C}$. Then, the supernatant was collected and total protein was determined using an ELISA kit. The protein levels of pro-inflammatory cytokines, such as tumor necrosis factor- $\alpha$ (TNF- $\alpha)$, interleukin $1-\beta$ (IL-1 $\beta$ ) and interleukin 6 (IL-6), were quantified using specific ELISA kits for rat cytokines according to the manufacturers' instructions (TNF- $\alpha$, IL-1 $\beta$ and IL-6 from LKCX Biotechnology, Beijing, China). Absorbance at $450 \mathrm{~nm}$ was measured on an enzyme-linked immunometric meter (BioTek Instruments Inc., Winooski, VT, USA). All samples, standards, and positive controls were run in duplicate so that all tissue could be run on a single plate, reducing a potential source of variability. Cytokine protein concentrations were calculated by the standard curve and expressed as nanograms per liter.

\section{Statistical analysis}

Statistical analysis was performed using SPSS Statistics, version 24.0 (IBM Corporation, Armonk, NY, USA). For the BBB score, we used one-way ANOVAs with Fisher's 
LSD post-test and paired $t$-tests; for other assays, one-way ANOVAs with Fisher's LSD post-test or Dunnett's T3 test were used in data analysis. Data were presented as mean \pm SEM, and differences were reported as statistically significant at $P<0.05$.

\section{Results}

\section{Neurofunctional outcome}

The motor function of all rats was normal (BBB Scale $=21$ ) pre-operative (Figure 2). At 1 day after SCI, all animals in the SCI group were completely paraplegic, while rats in the sham operation group had a moderate level of BBB scores. In the following days, a slow recovery of hind limb locomotion, characterized by slight movement in one or two joints, was observed in both groups. After 28 days of recovery, rats in the sham operation group had returned to normal. However, the BBB scores of both sides were still lower in the SCI group than ones in the sham operation group at day 28 post-operative $(P<0.001)$. Similarly, the BBB scores of both sides in other time points were significantly lower than the ones in the sham operation group $(P<0.001)$.

\section{Myelination of the spinal cord after SCl presented an anti-parabolic trend}

Representative images of LFB staining are shown in Figure 3. Myelin vacuolation after SCI was obvious, and myelination of the spinal cord showed an anti-parabolic trend. In other

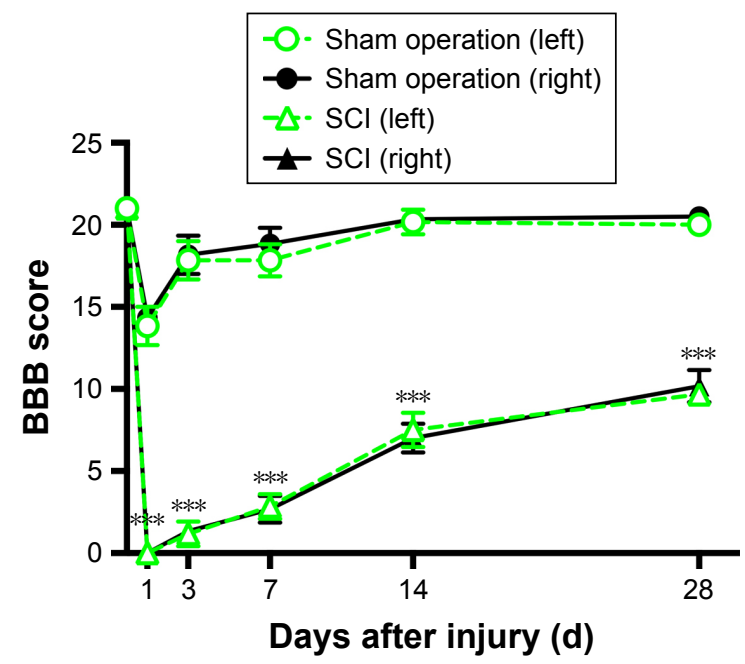

Figure 2 Line chart of the BBB scores of rats in the $\mathrm{SCl}$ and sham operation groups.

Note: The BBB scores of both sides were significantly lower in the $\mathrm{SCl}$ group than the ones in the sham operation group at days I, 3, 7, I4, and 28 post-operative $* * *(P<0.001, \mathrm{n}=108)$.

Abbreviations: $\mathrm{SCl}$, spinal cord injury; $\mathrm{BBB}$, Basso, Beattie, and Bresnahan Locomotor Rating Scale.
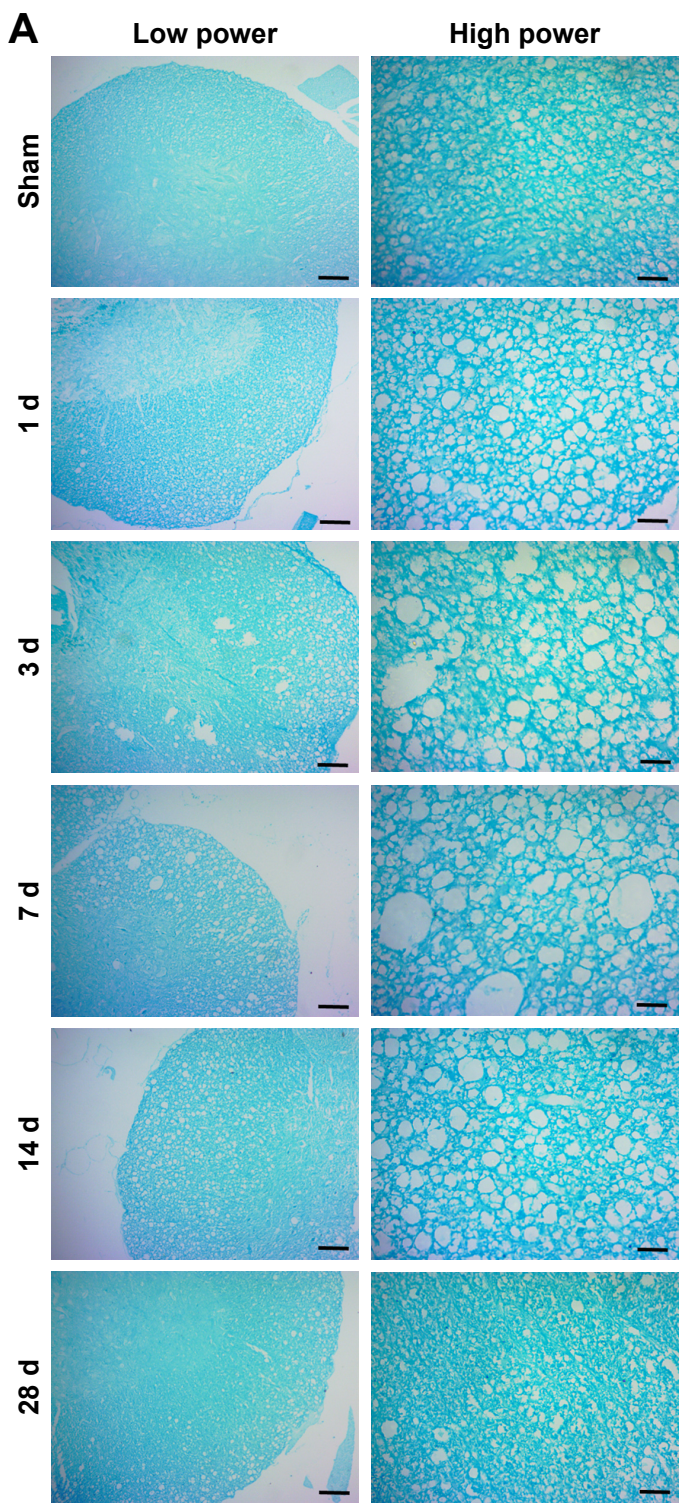

B

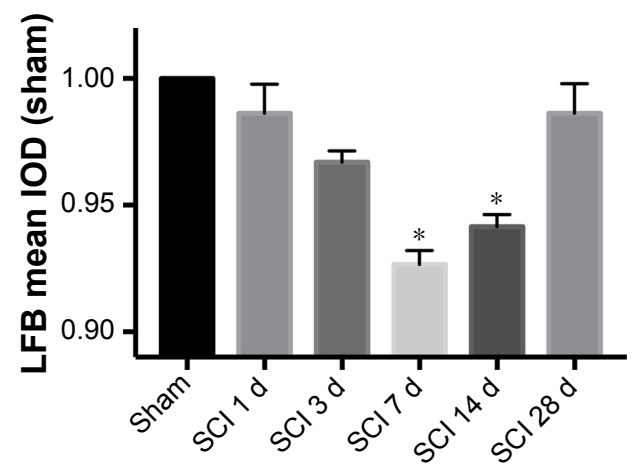

Figure 3 Myelination of the spinal cord after $\mathrm{SCl}$ investigated through staining of LFB.

Notes: (A) Representative images of LFB staining in the sham group and I, 3, 7, I4, and 28 days after $\mathrm{SCl}$. The left side of the figure was low-power field $(100 \times)$ and the right one was high-power field $(400 \times)$. Scale bar $=100 \mu \mathrm{m}$ (left column) and scale bar $=25 \mu \mathrm{m}$ (right column). (B) Bar graphs of myelination in different groups. It was progressively aggravated during the acute period, reached the trough at day 7 post injury $*(P<0.05, n=36)$, and then gradually recovered to close to the level of the sham operation group at day 28 post-operative. $* P<0.05$ compared with sham group.

Abbreviations: $\mathrm{d}$, days; $\mathrm{SCl}$, spinal cord injury; LFB, Luxol fast blue. 
words, it was progressively aggravated during the acute period, reached the trough at day 7 post injury $(P<0.05)$, and then gradually recovered to close to the level of the sham operation group at day 28 post-operative.

\section{Level of oligodendroglia was contra- parabola after $\mathrm{SCl}$}

The level of MBP and LPL expression may reflect the degree of injury of oligodendrocytes after SCI. An equal contra-parabola trend after SCI was found in MBP and LPL stainings. As shown in Figures 4 and 5, the mean IODs of both MBP and LPL declined during the acute period, reached the lowest level at day 7 after SCI $(P<0.001)$, and then gradually rose during 28 days post-operative. The protein expression of MBP protein in the six groups was detected using Western blotting analyses. As shown in Figure 6, MBP declined firstly, reached the lowest level at day 7 after SCI $(P<0.001)$, and gradually rose during 28 days post-operative $(P<0.001)$.

\section{The microglial activation appeared a tendency of parabolic line after $\mathrm{SCl}$}

In rats, activated microglia that have reached the phagocytic stage express ED-1, a lysosomal antigen expressed in phagocytes, while microglia involved in immune-mediated responses upregulate MHC-II expression. Representative images of double immunofluorescence staining are shown in Figure 7A. A marked parabolic trend is shown in Figure 7B concerning the mean number of the activated microglial involved in immune-mediated responses. ED- $1^{+} \mathrm{MHC}-\mathrm{II}^{+}$ cells at day 7 after SCI were significantly more than ones of the sham operation group $(P<0.001)$. The number of double positive cells decreased at 4 weeks after injury, but it was still higher than that of the sham operation group $(P<0.05)$.

\section{Iron and glutamate concentration were remarkably higher while ATP and potassium concentration were significantly lower at day 7 after $\mathrm{SCl}$}

After injury, damaged cells can be cracked or secreted to produce many factors such as potassium, ATP, iron, and glutamate. In the tissues around the injury site, the above factors were investigated. Interestingly, compared with the sham operation group, both iron and glutamate concentration began to rise significantly at 1 day (Figure $8 \mathrm{~A}, P<0.01$; and Figure $8 \mathrm{~B}, P<0.001$ ), peaked at 7 days (Figure $8 \mathrm{~A}$, $P<0.01$; and Figure $8 \mathrm{~B}, P<0.001$ ), and then declined until

\section{A
$\frac{\varepsilon}{5}$
$\frac{5}{\infty}$}
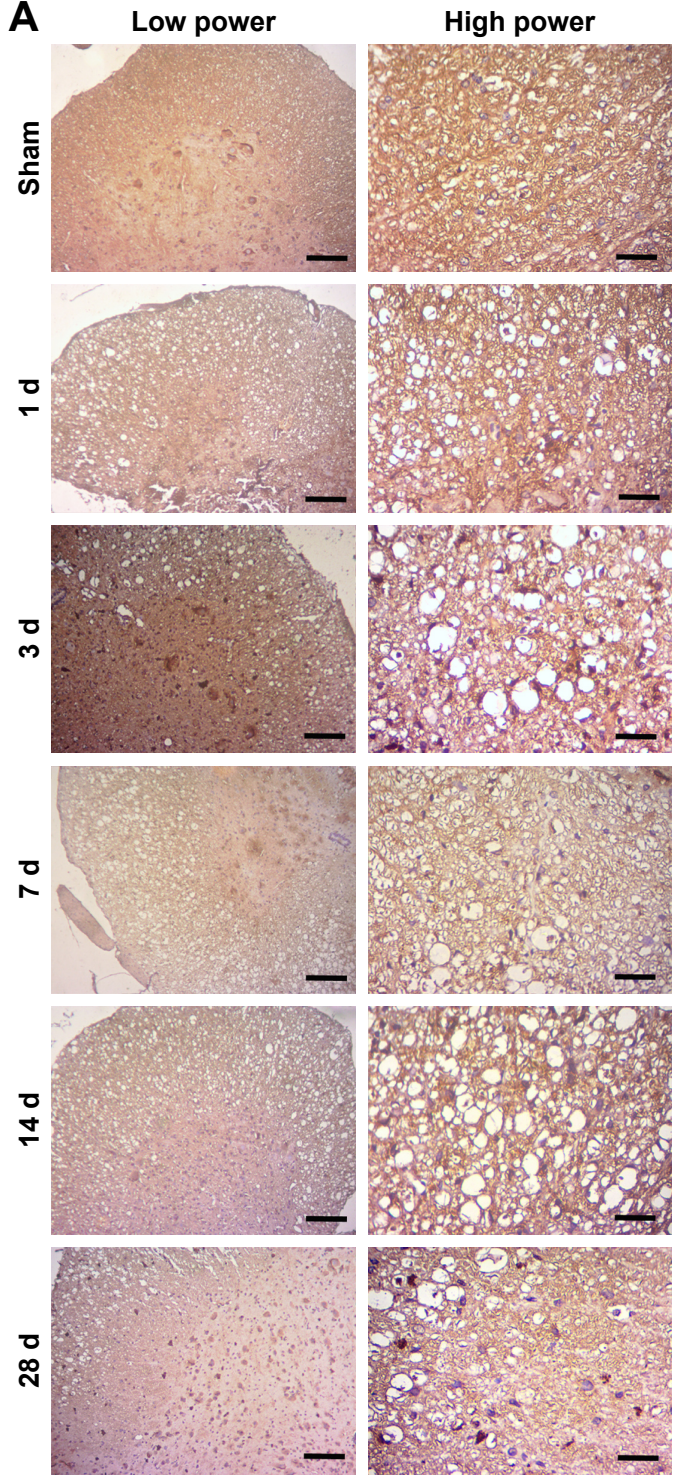

B

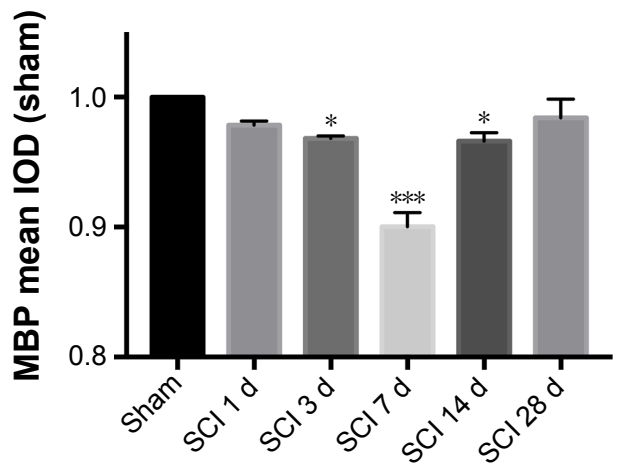

Figure 4 Immunohistochemistry of $\mathrm{MBP}$ in the $\mathrm{SCl}$ and sham operation groups. Notes: (A) Representative images of MBP staining in the sham group and SCl group I, 3, 7, 14, and 28 days post injury. The images on the left are a low field of vision $(100 \times)$, while a high-power view $(400 \times)$ is on the right. Scale bar $=100 \mu \mathrm{m}$ (left column) and scale bar $=25 \mu \mathrm{m}$ (right column). (B) Bar graphs of the degree of injury of oligodendroglia in different groups. The mean IODs of MBP declined during the acute period, reached the lowest level at day seven after $\mathrm{SCl} * * *(P<0.00 \mathrm{I}, \mathrm{n}=36)$, and then gradually rose during 28 days post-operative. The mean IODs of MBP at day 3 and 14 post-operative were lower than ones in the sham operation group $*(P<0.05, n=36) . * P<0.05$, and $* * * P<0.001$ compared with the sham group.

Abbreviations: d, days; MBP, Myelin basic protein; $\mathrm{SCl}$, spinal cord injury; IOD, integrated optical density. 
A
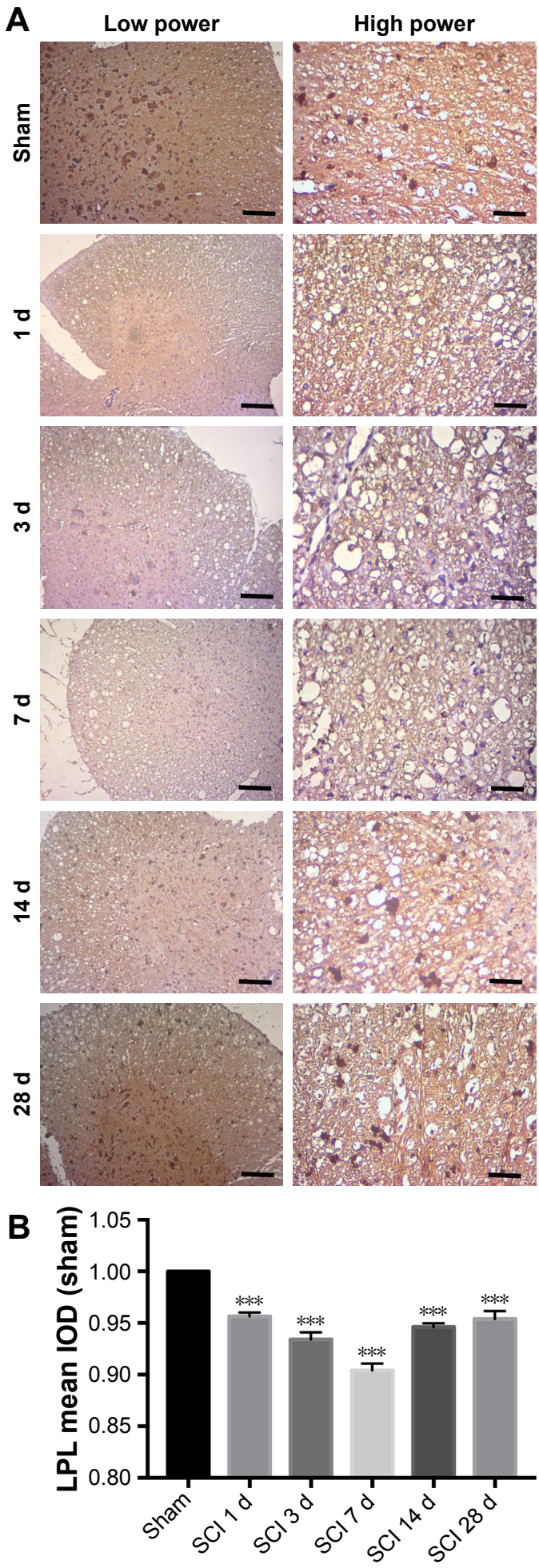

Figure 5 Immunohistochemistry of LPL in tissue from different time points after $\mathrm{SCl}$ and sham operation groups.

Notes: (A) Representative images of LPL staining in the sham group and $\mathrm{SCl}$ group $\mathrm{I}, 3,7,14$, and 28 days post injury. On the left are the figures under a low field of vision $(100 \times)$, while on the right are the ones under a high-power view $(400 \times)$. Scale bar $=100 \mu \mathrm{m}$ (left column) and scale bar $=25 \mu \mathrm{m}$ (right column). (B) Bar graphs of the degree of injury of oligodendroglia in different groups. The mean IODs of LPL gradually declined during the acute period, reached the lowest level at day 7 after $\mathrm{SCl} * * *(P<0.001, \mathrm{n}=36)$, and then began to rise afterward during 28 days postoperative. ${ }^{*} * * P<0.001$ compared with sham group.

Abbreviations: $d$, days; LPL, rabbit anti-Plastin L; IOD, integrated optical density; $\mathrm{SCl}$, spinal cord injury.
A

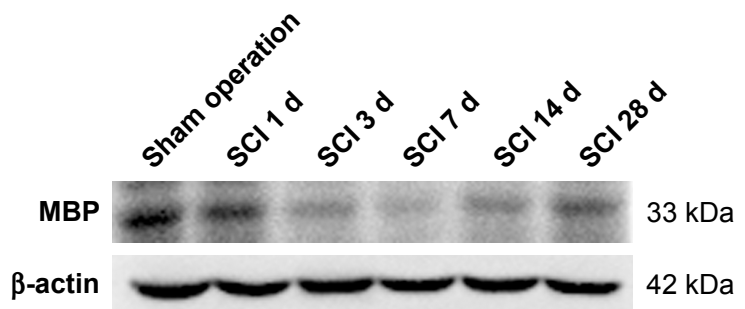

B

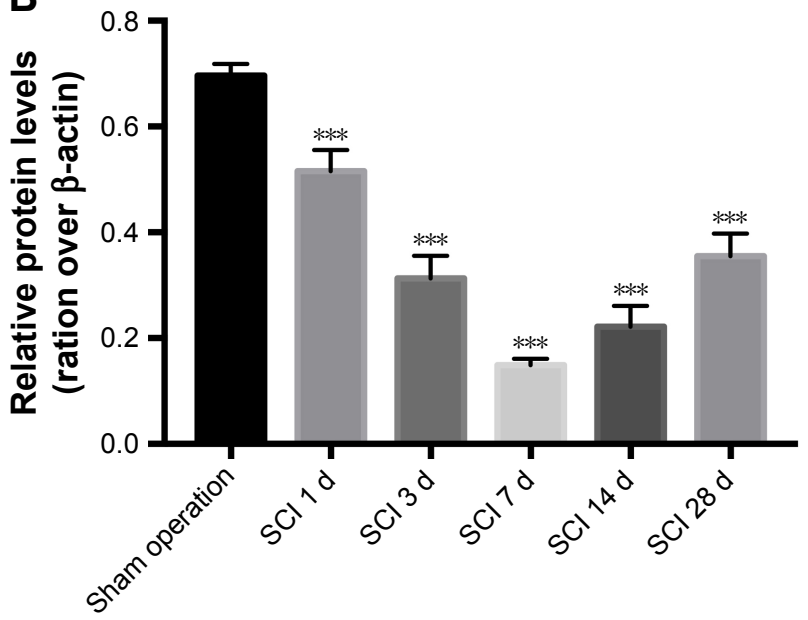

Figure 6 The protein expression of MBP was detected using Western blotting analyses.

Notes: (A) Representative images of Western blot in the sham group and $\mathrm{SCl}$ group I, 3, 7, 14, and 28 days post injury. (B) Bar graphs of the level of MBP protein in different groups. The ratio of MBP and $\beta$-actin declined firstly, reached the lowest level at day 7 after $\mathrm{SCl} * * *(P<0.001, n=36)$, and then rose during 28 days post-operative $* * *(P<0.001, n=36)$. *** $P<0.001$ compared with the sham group.

Abbreviations: $\mathrm{d}$, days; MBP, Myelin basic protein; $\mathrm{SCl}$, spinal cord injury.

day 28 post-operative (Figure $8 \mathrm{~A}, P<0.01$; and Figure $8 \mathrm{~B}$, $P<0.001)$.

Conversely, both ATP and potassium concentrations began to decline significantly at 1 day (Figure $8 \mathrm{C}, P<0.001$; and Figure $8 \mathrm{D}, P<0.001)$, reached a low valley at 7 days (Figure $8 \mathrm{C}, P<0.001$; and Figure $8 \mathrm{D}, P<0.001$ ), and then gradually recovered. But at day 28 post-operative, they still were below that of the sham operation group (Figure 8C, $P<0.001$; and Figure 8D, $P<0.001)$.

\section{Inflammatory response was the most severe at day 7 after $\mathrm{SCl}$}

Activated microglia responding to SCI can produce proinflammatory cytokines such as TNF- $\alpha$, IL-1 $1 \beta$ and IL-6. Levels of the above pro-inflammatory cytokines increased significantly at 1 day (Figure $9 \mathrm{~A}-\mathrm{C}, P<0.001$ ), peaked at 7 days (Figure 9A-C $P<0.001$ ), and gradually decreased. After 4 weeks, the three cytokine concentrations remained nearly 3 -fold higher than the ones of the sham operation group (Figure 9A-C, $P<0.001$ ). 


\section{A}

Low power

High power
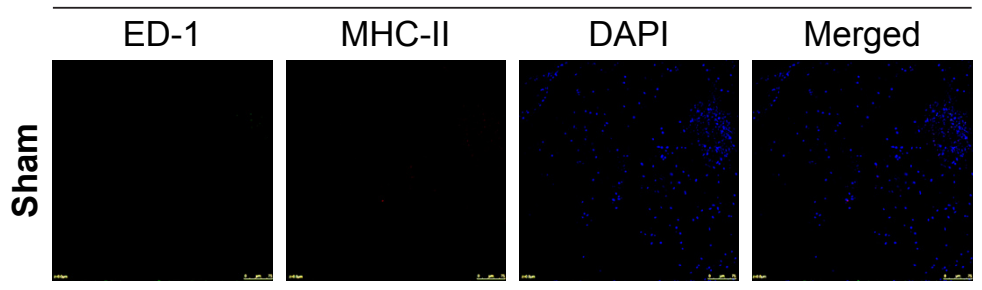

Merged
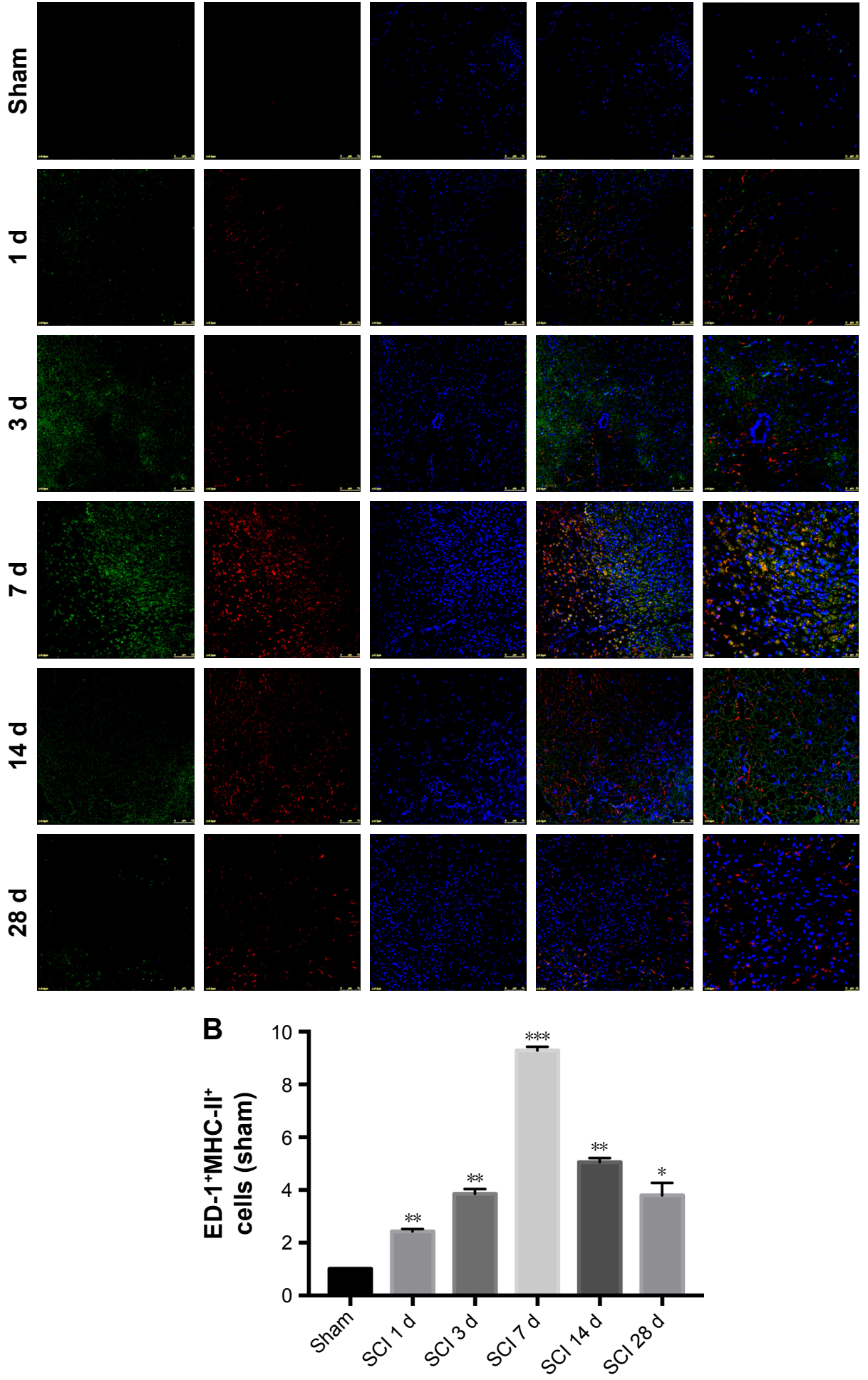

Figure 7 Double staining of ED-I and MHC-II were used to investigate the microglial activation.

Notes: (A) Representative ED-I/MHC-II/DAPI photomicrographs of the spinal cord tissue in the different groups. In addition to pictures of the right-hand column which are a high-power field $(400 \times)$, the other columns are low-power field $(200 \times)$. Fluorescence colors: MHC-II: red, ED-I: green, and DAPI: blue. Scale bar $=50 \mu \mathrm{m}$ (left three columns) and scale bar $=25 \mu \mathrm{m}$ (right-hand column). (B) Bar graphs of the mean number of the activated microglial involved in immune-mediated responses in the different groups. ED- $\mathrm{I}^{+} \mathrm{MHC}-\mathrm{II}^{+}$cells at day 7 after SCl were significantly more than ones of the sham operation group $* * *(P<0.00 \mathrm{I}, \mathrm{n}=36)$. The number of double positive cells decreased at 4 weeks after injury, but it was still higher than that of sham operation group $*(P<0.05, n=36) . * P<0.05, * * P<0.01$, and $* * * P<0.00$ I compared with sham group. Abbreviations: d, days; DAPI, 4',6-diamidino-2-phenylindole; SCl, spinal cord injury. 
A

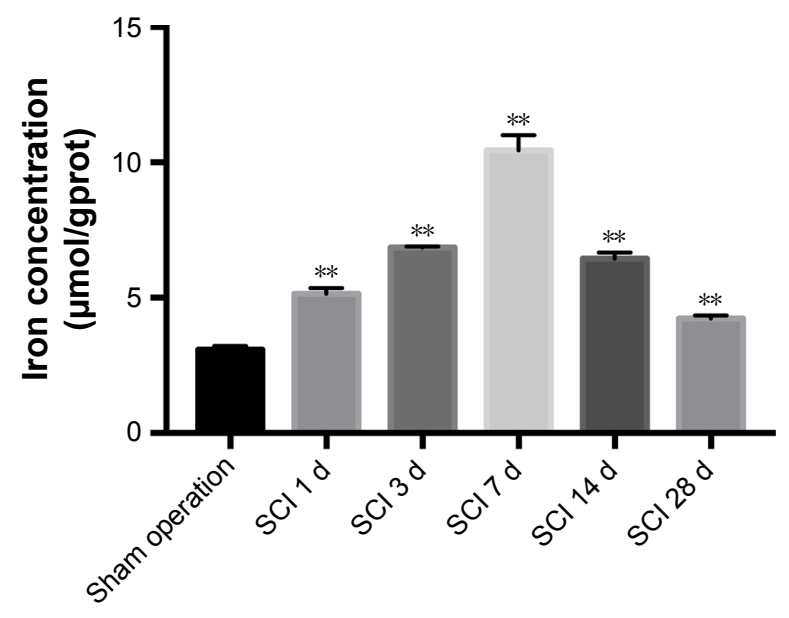

C

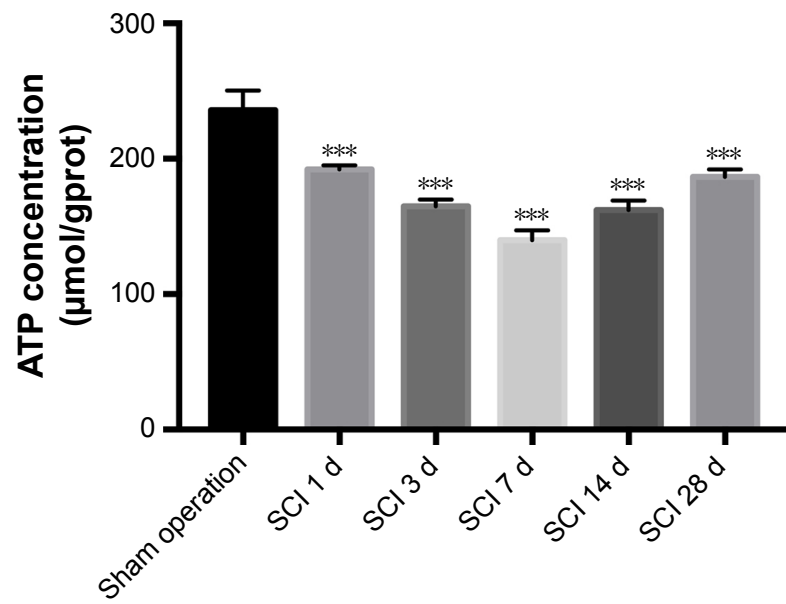

B

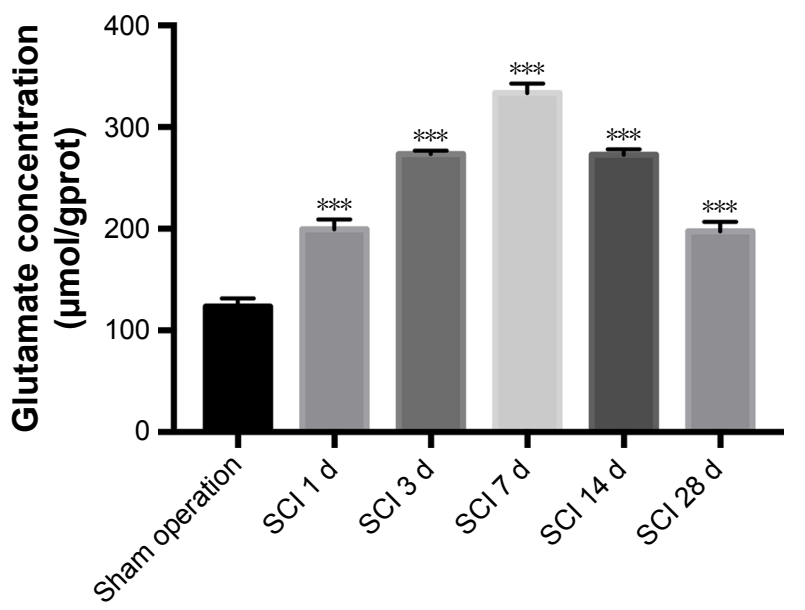

D

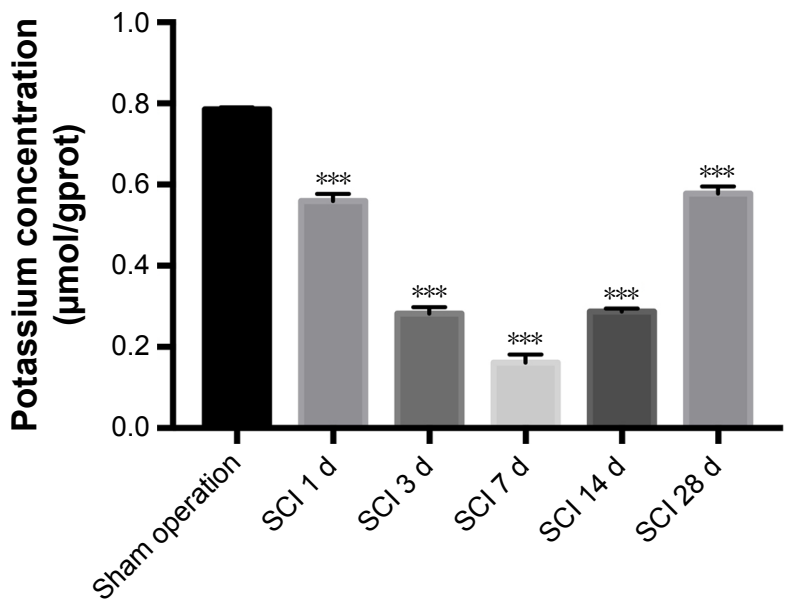

Figure $8 \mathrm{Bar}$ graphs of iron, glutamate, ATP and potassium concentrations in the tissues around the injury site were compared in the different groups.

Notes: (A, B) Compared with the sham operation group, both iron and glutamate concentration began to rise significantly at I day $(* * \mathrm{p}<0.0 \mathrm{I}$, and $* * * \mathrm{P}<0.00 \mathrm{I}, \mathrm{n}=36)$, peaked at 7 days $(* * \mathrm{P}<0.0 \mathrm{I}$, and $* * * \mathrm{P}<0.00 \mathrm{I}, \mathrm{n}=36)$, and then declined until day 28 post-operative $(* * \mathrm{P}<0.0 \mathrm{I}$, and $* * * \mathrm{P}<0.00 \mathrm{I}, \mathrm{n}=36)$. (C, D) Conversely, both $\mathrm{ATP}$ and potassium concentration began to decline significantly at I day $(* * * P<0.00 \mathrm{I}$, and $* * * P<0.00 \mathrm{I}, \mathrm{n}=36)$, reached a low valley at 7 days $(* * * P<0.00 \mathrm{I}$, and $* * * P<0.00 \mathrm{I}, \mathrm{n}=36)$, and then gradually recovered. But at day 28 post-operative, they still were below that of the sham operation group ( $* * * P<0.00 \mathrm{I}$, and $* * * P<0.00 \mathrm{I}, \mathrm{n}=36$ ). $* * P<0.0 \mathrm{I}$, and $* * * P<0.001$ compared with sham group.

Abbreviations: $d$, days; $\mathrm{SCl}$, spinal cord injury.

\section{Discussion}

It is considered that SCI-inducing factors directly affect oligodendroglia cells and the myelin sheath, leading to primary injury of both, while microglia activation plays an important role in secondary injury after SCI., ${ }^{5,22}$ Secondary injury is the research emphasis owing to primary injury of non-intervention. Although the above factors have been studied for decades, the exact time-course profiles of microglial activation and the specific factors are varied and far from clear using different SCI models. ${ }^{8,23,24}$ To clarify the microglial activation profile and associated factors, a rat compressive SCI model was employed in this study.

Currently recognized pathophysiology mechanisms following SCI mainly include oligodendroglial apoptosis along with degenerating axons in rats. A previous report demonstrated that many oligodendroglia apoptosis had been observed a day after SCI and lasted for more than 3 weeks, which eventually led to the occurrence of chronic demyelination. ${ }^{8}, 25$ Severe chronic demyelination not only causes secondary injury of axons but also affects the morphology, structure and function of the normal myelin sheath characterized by "waterfall enlargement." ${ }^{26,27}$ Studies in our laboratory and by Professor Totoiu in the USA have found clear chronic demyelinating changes in different animal SCI models. ${ }^{27}$ Similarly, this study detected obvious myelin vacuolation and injury of oligodendrocytes after SCI. Furthermore, the level of myelination and oligodendroglia were contra-parabola after SCI. Damage 
A

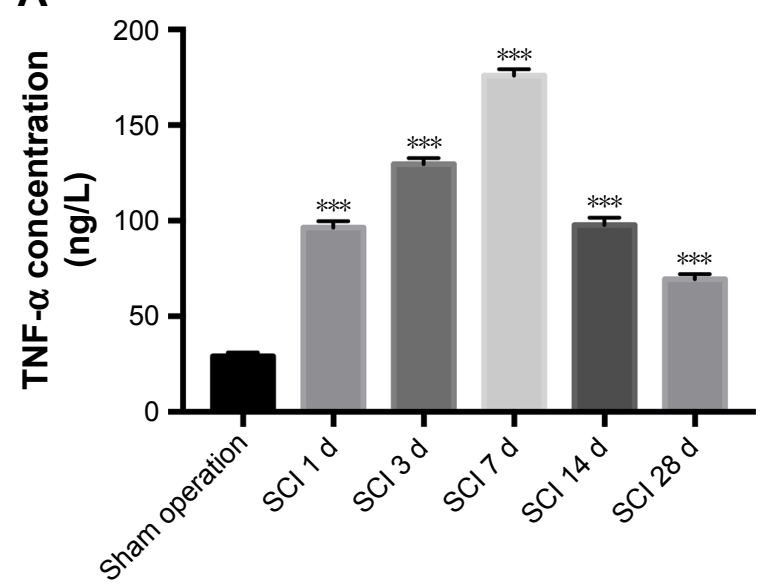

B

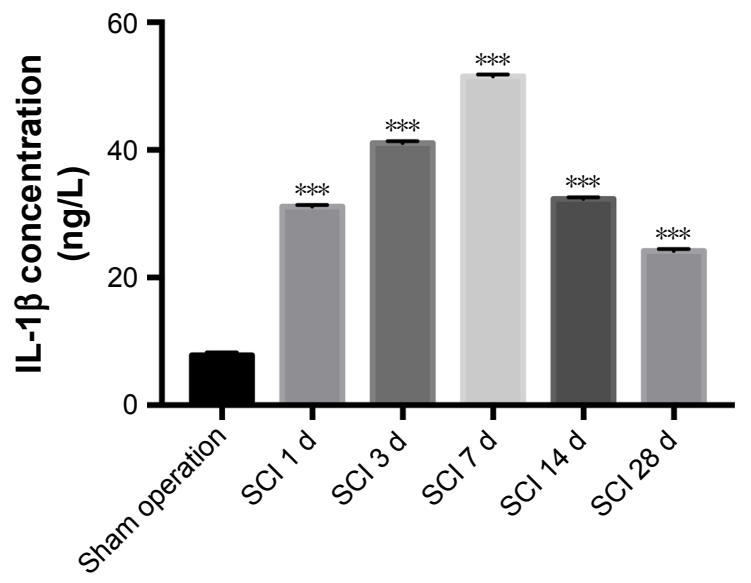

C

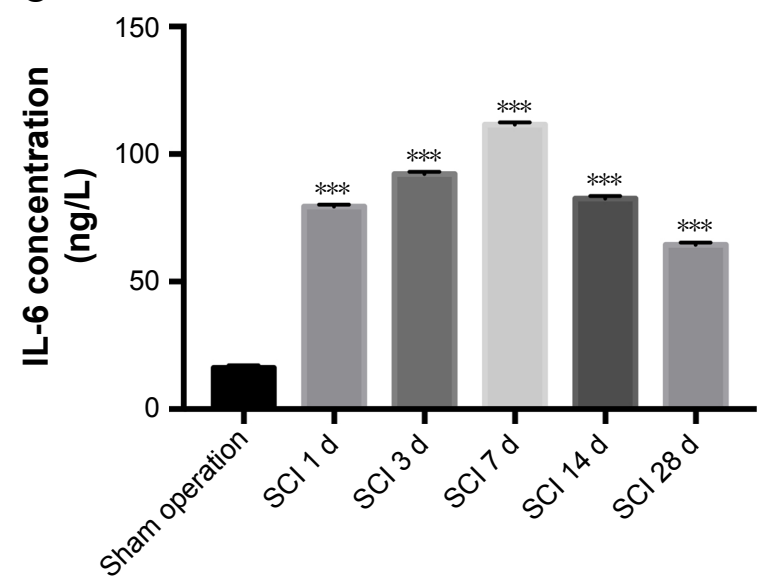

Figure 9 Bar graphs of the protein levels of pro-inflammatory cytokines, such as TNF- $\alpha$, IL-I $\beta$, and IL-6, were compared in the SCl and sham operation groups. Notes: $(\mathbf{A}-\mathbf{C})$ Level of above pro-inflammatory cytokines increased significantly at I day $* * *(P<0.00 \mathrm{I}, \mathrm{n}=36)$, peaked at 7 days $* * *(P<0.00 \mathrm{I}, \mathrm{n}=36)$, and gradually decreased. After 4 weeks, the three cytokine concentrations remained nearly 3 -fold higher than ones of the sham operation group $* * *(P<0.00 I, n=36)$. $* * * P<0.00 I$ compared with sham group.

Abbreviations: $\mathrm{d}$, days; $\mathrm{SCl}$, spinal cord injury.

was clear at day 1 post-operative, gradually deteriorated, peaked at day 7 , and ameliorated gently thereafter. Those deteriorations of injury lasting for 7 days may lie in complex wound factors which accelerate the second course caused by malignant positive feedback mechanisms under the pathological conditions like acute pancreatitis.

Microglia are such key factors which may cause the 7-day deterioration of injury. Microglial cells are the resident tissue macrophages in the CNS and are on constant surveillance for perturbations resulting from injury. ${ }^{28}$ However, different microglial activation profiles have been found in varied studies. ${ }^{8,11,22}$ The results of glial cell quantification in the contusion rat SCI model showed a significant increase in glial cell density percentage at day 2 as compared to other days, and the highest increase in ED-1 immunoreactive cells was observed at day $2(23.15 \%)$ post-injury. ${ }^{8}$ Another study demonstrated that microglia had peaked at day 7 and then decreased at day 14 post-SCI to levels comparable to that observed at days 1 and 3 following spinal cord compression injury in the lys-egfp-ki mouse model. ${ }^{14}$ Accurate time-course profiles of microglial activation have not been demonstrated hence. In our rat compressive SCI model, the activated microglia involved in immune-mediated responses increased dramatically after injury, reaching the peak at day 7 , and then dropped subsequently. Our results are similar to those of previous results. Previous studies found that microglia had at least two different spatial and temporal patterns of activation after compressive SCI. One was rapid and most likely involved the blood-borne complement activating system. The other accompanied Wallerian degeneration and was independent of the blood-borne complement system. Varied activated patterns may lead to diverse microglial characters.

There is evidence that microglia contribute to secondary tissue damage in injury of the CNS. ${ }^{29,30}$ Activated microglia 
may demonstrate detrimental effects to impair the remaining oligodendroglia and myelin through the following ways. ${ }^{3}$ First of all, they produce various pro-inflammatory cytokines, proteases, and other factors that are cytotoxic in the injured spinal cord. ${ }^{31}$ IL-1, IL-6, and TNF are the most important pro-inflammatory cytokines and often act in conjunction. ${ }^{17}$ In our test, the protein levels of TNF- $\alpha$, IL-1 $\beta$, and IL- 6 peaked at 7 days which represented the most severe inflammatory response. Interestingly at this point, demyelination and microglial activation were also most serious within 4 weeks. This phenomenon indirectly reflected the increased inflammatory response after microglia activation post SCI. Similar findings demonstrated that microglia activation had resulted in inflammatory response in rats following SCI, and D1-3-n-butylphthalide may have neuroprotective and anti-inflammatory properties in the treatment of SCI by inhibiting the activation of microglia via TLR4/NF-kappaB signaling. ${ }^{20} \mathrm{Next}$, astrocytes were activated to further enhance the inflammation response. ${ }^{32,33}$ The finding showed after SCI, reactive astrocytes had undergone M1 microglia-induced necroptosis, partially through TLR/ MyD88 signaling, and suggested that inhibiting astrocytic necroptosis may be beneficial for preventing secondary SCI. ${ }^{33}$ The third one was to produce large amounts of hydrogen peroxide and superoxide anion for the generation of oxidative stress. ${ }^{34,35}$ The reduced activation of microglia after 7 days post injury also implied lessened injury of oligodendrocytes, demyelination and inflammatory response. After 4 weeks, approximate epistrophy of oligodendrocytes and myelination did not have matching neurofunctional outcomes and inflammatory responses. The still poor hind limb locomotion may be due to irreversible damage of neurons and regenerated myelin with incomplete function. We also speculate that probably the inflammatory response can be induced by other elements other than activation of microglia. Recent findings indicated that microglia may sculpt the structure of the CNS, refine neuronal circuitry and network connectivity, and contribute to plasticity rather than their detrimental effects. ${ }^{12,36}$ They function to maintain normal homeostasis and deal with issues of cell death in a manner that minimizes neuro-inflammation under different environments. ${ }^{3,37}$ In our SCI model, activated microglia may play a proinflammatory response primarily.

The activation of microglial has been shown to be influenced by many factors after injury. Except for injury, damaged or mutant oligodendroglia are likely to be another reason for the activation of microglial in our experimental SCI model. Zhang et al's research suggested that as an internal environment detector, microglial cells did not express MHC-II at first and they had not been truly activated until many abnormal vesicles were generated in the remaining oligodendroglia. ${ }^{11}$ More direct evidence comes from a review published on Journal of Neuroscience: extracellular high concentration of glutamate can produce excitotoxic cytotoxicity to oligodendroglia and the myelin sheath. At certain concentrations, glutamate can induce microglial to activate, releasing TNF- $\alpha$ and IL-1, and express metabotropic glutamate receptors (mGluR1-8) and ionotropic glutamate receptors (NMDA, AMPA, etc.). ${ }^{16}$ In addition to microglial cells, the axon and the remaining oligodendroglia were also the main source of glutamate. Interestingly in our study, iron and glutamate content reached the peak at day 7 after SCI, the most significant time point for the activation of microglial. SCI may cause intracranial iron overload through the NOS-iron-responsive element/IRP pathway, resulting in central pain mediated by the oxidative stress response. ${ }^{38}$ Other findings showed that high intracellular iron accumulation in macrophages had induced TNF expression. ${ }^{17}$ Thus, iron accumulation after SCI may also contribute to the activation of microglial. However, both ATP and potassium reached a low valley at 7 days post-operative. Release of ATP induced the outgrowth of microglial processes to the site of injury. Both transmitter systems, NO and ATP, were active on spinal microglia. When ambient ATP decreased in the extracellular environment, local NO application elicited less process attractions which attenuated the activation of microglia finally. ${ }^{18}$ Growing evidences indicate that the voltage-gated potassium channels have participated in neuronal damage and microglia function. ${ }^{19}$ Potassium channels facilitate the refilling of intracellular $\mathrm{Ca}^{2+}$ stores following ATP or UTP activation of metabotropic P2Y-receptors, thus maintaining high $\mathrm{Ca}^{2+}$ and timing of intracellular signaling events important for microglia activation and proliferation. ${ }^{39}$ Hence, we conclude that reduced potassium in the tissues around injury site is also vital for the activation of microglia after SCI. Our experiment demonstrated an increment in iron and glutamate content while a decrement in ATP and potassium post SCI. Based on the above clues and our results, such increments of glutamate and iron in particular time could potentially lead to consistent microglia activation.

\section{Conclusion}

Although the microglia roles after SCI have been studied for decades, the exact effect, time-course profile and factors are far from clear, resulting in a lack of targeted treatment and poor quality of life. Microglia activation plays 
an important role in second injuries post SCI. Our results showed a tendency of parabolic line in microglia activation after SCI, in which the seventh day was the peak with the most severe injury to oligodendroglia cells and the myelin sheath. Meanwhile, high iron and glutamate concentration may be released by damaged oligodendroglia and contribute to the activation of microglial after our experimental SCI model in rats. Therefore, a more in-depth study exploring an objective and feasible treatment method aimed at microglia and associated factors may possibly improve long-term neurological function of SCI and have greater medical benefits.

\section{Acknowledgment}

This work was funded by grants from the National Natural Science Foundation of China (no 81371356), the Youth Training Project (no 16QNP042), and the Jinling Hospital of Nanjing (no 2016017).

\section{Disclosure}

The authors report no conflicts of interest in this work.

\section{References}

1. Ning GZ, Wu Q, Li YL, Feng SQ. Epidemiology of traumatic spinal cord injury in Asia: a systematic review. J Spinal Cord Med. 2012; 35(4):229-239.

2. Lee BB, Cripps RA, Fitzharris M, Wing PC. The global map for traumatic spinal cord injury epidemiology: update 2011, global incidence rate. Spinal Cord. 2014;52(2):110-116.

3. Alizadeh A, Karimi-Abdolrezaee S. Microenvironmental regulation of oligodendrocyte replacement and remyelination in spinal cord injury. J Physiol. 2016;594(13):3539-3552.

4. Hu HZ, Granger N, Jeffery ND. Pathophysiology, clinical importance, and management of neurogenic lower urinary tract dysfunction caused by suprasacral spinal cord injury. J Vet Intern Med. 2016;30(5): $1575-1588$.

5. Hayta E, Elden H. Acute spinal cord injury: A review of pathophysiology and potential of non-steroidal anti-inflammatory drugs for pharmacological intervention. J Chem Neuroanat. 2018;87:25-31.

6. Eldahan KC, Rabchevsky AG. Autonomic dysreflexia after spinal cord injury: Systemic pathophysiology and methods of management. Auton Neurosci. 2018;209:59-70.

7. Ma L, Yu HJ, Gan SW, et al. p53-Mediated oligodendrocyte apoptosis initiates demyelination after compressed spinal cord injury by enhancing ER-mitochondria interaction and E2F1 expression. Neurosci Lett. 2017;644:55-61.

8. Abdanipour A, Tiraihi T, Taheri T, Kazemi H. Microglial activation in rat experimental spinal cord injury model. Iran Biomed J. 2013;17(4):214-220.

9. Wang W, Huang X, Li J, et al. Methane suppresses microglial activation related to oxidative, inflammatory, and apoptotic injury during spinal cord injury in rats. Oxid Med Cell Longev. Epub 2017 Jun 2017.

10. von Leden RE, Khayrullina G, Moritz KE, Byrnes KR. Age exacerbates microglial activation, oxidative stress, inflammatory and NOX2 gene expression, and delays functional recovery in a middle-aged rodent model of spinal cord injury. J Neuroinflammation. 2017;14(1): 161.
11. Zhang SC, Goetz BD, Carré JL, Duncan ID. Reactive microglia in dysmyelination and demyelination. Glia. 2001;34(2):101-109.

12. David S, Kroner A. Repertoire of microglial and macrophage responses after spinal cord injury. Nat Rev Neurosci. 2011;12(7):388-399.

13. Magaki SD, Williams CK, Vinters HV. Glial function (and dysfunction) in the normal and ischemic brain. Neuropharmacology. 2018;134(Pt B): 218-225.

14. Thawer SG, Mawhinney L, Chadwick K, et al. Temporal changes in monocyte and macrophage subsets and microglial macrophages following spinal cord injury in the Lys-Egfp-ki mouse model. JNeuroimmunol. 2013;261(1-2):7-20.

15. Tateda S, Kanno H, Ozawa H, et al. Rapamycin suppresses microglial activation and reduces the development of neuropathic pain after spinal cord injury. J Orthop Res. 2017;35(1):93-103.

16. Fern R, Möller T. Rapid ischemic cell death in immature oligodendrocytes: a fatal glutamate release feedback loop. J Neurosci. 2000;20(1):34-42.

17. Kroner A, Greenhalgh AD, Zarruk JG, Passos dos Santos R, Gaestel M, David S. TNF and increased intracellular iron alter macrophage polarization to a detrimental M1 phenotype in the injured spinal cord. Neuron. 2014;83(5):1098-1116.

18. Dibaj P, Nadrigny F, Steffens H, et al. NO mediates microglial response to acute spinal cord injury under ATP control in vivo. Glia. 2010;58(9):1133-1144.

19. Wendt S, Maricos M, Vana N, et al. Changes in phagocytosis and potassium channel activity in microglia of $5 \times \mathrm{FAD}$ mice indicate alterations in purinergic signaling in a mouse model of Alzheimer's disease. Neurobiol Aging. 2017;58:41-53.

20. He Z, Zhou Y, Lin L, et al. Dl-3-n-butylphthalide attenuates acute inflammatory activation in rats with spinal cord injury by inhibiting microglial TLR4/NF-אB signalling. J Cell Mol Med. 2017;21(11): 3010-3022.

21. Smith PD, Bell MT, Puskas F, et al. Preservation of motor function after spinal cord ischemia and reperfusion injury through microglial inhibition. Ann Thorac Surg. 2013;95(5):1647-1653.

22. Wei Z, Yu D, Bi Y, Cao Y. A disintegrin and metalloprotease 17 promotes microglial cell survival via epidermal growth factor receptor signalling following spinal cord injury. Mol Med Rep. 2015;12(1):63-70.

23. Schwab JM, Frei E, Klusman I, Schnell L, Schwab ME, Schluesener HJ. AIF-1 expression defines a proliferating and alert microglial $/ \mathrm{mac}-$ rophage phenotype following spinal cord injury in rats. J Neuroimmunol. 2001;119(2):214-222.

24. Alluin O, Delivet-Mongrain H, Gauthier MK, Fehlings MG, Rossignol S, Karimi-Abdolrezaee S. Examination of the combined effects of chondroitinase $\mathrm{ABC}$, growth factors and locomotor training following compressive spinal cord injury on neuroanatomical plasticity and kinematics. PLoS One. 2014;9(10):e111072.

25. Redondo-Castro E, Hernández J, Mahy N, Navarro X. Phagocytic microglial phenotype induced by glibenclamide improves functional recovery but worsens hyperalgesia after spinal cord injury in adult rats. Eur J Neurosci. 2013;38(12):3786-3798.

26. Silver J, Schwab ME, Popovich PG. Central nervous system regenerative failure: role of oligodendrocytes, astrocytes, and microglia. Cold Spring Harb Perspect Biol. 2014;7(3):a020602.

27. Totoiu MO, Keirstead HS. Spinal cord injury is accompanied by chronic progressive demyelination. J Comp Neurol. 2005;486(4):373-383.

28. Bermudez S, Khayrullina G, Zhao Y, Byrnes KR. NADPH oxidase isoform expression is temporally regulated and may contribute to microglial/macrophage polarization after spinal cord injury. Mol Cell Neurosci. 2016;77:53-64.

29. Emmetsberger J, Tsirka SE. Microglial inhibitory factor (MIF/TKP) mitigates secondary damage following spinal cord injury. Neurobiol Dis. 2012;47(3):295-309.

30. Qu WS, Tian DS, Guo ZB, et al. Inhibition of EGFR/MAPK signaling reduces microglial inflammatory response and the associated secondary damage in rats after spinal cord injury. $J$ Neuroinflammation. 2012;9:178. 
31. Jung GY, Lee JY, Rhim H, Oh TH, Yune TY. An increase in voltagegated sodium channel current elicits microglial activation followed inflammatory responses in vitro and in vivo after spinal cord injury. Glia. 2013;61(11):1807-1821.

32. Goldshmit Y, Kanner S, Zacs M, et al. Rapamycin increases neuronal survival, reduces inflammation and astrocyte proliferation after spinal cord injury. Mol Cell Neurosci. 2015;68:82-91.

33. Fan H, Zhang K, Shan L, et al. Reactive astrocytes undergo M1 microglia/macrophages-induced necroptosis in spinal cord injury. Mol Neurodegener. 2016;11:14.

34. Santos-Nogueira E, López-Serrano C, Hernández J, et al. Activation of lysophosphatidic acid Receptor Type 1 contributes to pathophysiology of spinal cord injury. J Neurosci. 2015;35(28):10224-10235.

35. Wang S, Zhang T, Yang Z, et al. Heme oxygenase-1 protects spinal cord neurons from hydrogen peroxide-induced apoptosis via suppression of Cdc42/MLK3/MKK7/JNK3 signaling. Apoptosis. 2017;22(3): $449-462$.
36. Loane DJ, Kumar A. Microglia in the TBI brain: the good, the bad, and the dysregulated. Exp Neurol. 2016;275(Pt 3):316-327.

37. Salter MW, Beggs S. Sublime microglia: expanding roles for the guardians of the CNS. Cell. 2014;158(1):15-24.

38. Meng FX, Hou JM, Sun TS. Effect of oxidative stress induced by intracranial iron overload on central pain after spinal cord injury. J Orthop Surg Res. 2017;12(1):24.

39. Nguyen HM, Blomster LV, Christophersen P, Wulff H. Potassium channel expression and function in microglia: plasticity and possible species variations. Channels. 2017;11(4):305-315.
Neuropsychiatric Disease and Treatment

\section{Publish your work in this journal}

Neuropsychiatric Disease and Treatment is an international, peerreviewed journal of clinical therapeutics and pharmacology focusing on concise rapid reporting of clinical or pre-clinical studies on a range of neuropsychiatric and neurological disorders. This journa is indexed on PubMed Central, the 'PsycINFO' database and CAS,

\section{Dovepress}

and is the official journal of The International Neuropsychiatric Association (INA). The manuscript management system is completely online and includes a very quick and fair peer-review system, which is all easy to use. Visit http://www.dovepress.com/testimonials.php to read real quotes from published authors.

Submit your manuscript here: http://www.dovepress.com/neuropsychiatric-disease-and-treatment-journal 